\title{
On Numerical Types of Algebraic Curves on Rational Surfaces
}

\author{
Osamu MATSUDA \\ Gakushuin University \\ (Communicated by K. Akao)
}

\section{Introduction.}

The purpose of this paper is to study numerical properties of algebraic curves on rational surfaces. Here by curves and surfaces we mean projective irreducible varieties of dimension 1 and 2 , respectively, which are defined over the field of complex numbers. We shall study pairs $(S, D)$ of projective non-singular rational surfaces $S$ and curves $D$ on $S$.

First, we recall some basic notions and results concerning birational geometry of pairs. Let $\Sigma_{b}$ denote a $\mathbf{P}^{1}$-bundle over $\mathbf{P}^{1}$ that has a minimal section $\Delta_{\infty}$ with $\Delta_{\infty}^{2}=-b \leq 0$. Furthermore, let $C$ be a curve on $\Sigma_{b}$. The Picard group of $\Sigma_{b}$ is generated by the section $\Delta_{\infty}$ and a fiber $F_{u}=\rho^{-1}(u)$ of the $\mathbf{P}^{1}$-bundle $\Sigma_{b}$ where $\rho$ is the projection (cf. [H1, p. 370, Proposition 2.3]). Then $C \sim \sigma \Delta_{\infty}+e F_{u}$ for some integers $\sigma$ and $e$. Here the symbol $\sim$ indicates linear equivalence between divisors. If $b \geq 1,(\sigma, e)$ is uniquely determined. But when $b=0$, the $\Sigma_{0}$ has two $\mathbf{P}^{1}$ - bundle structures. In this case, we may assume that $e \geq \sigma$. Then $(\sigma, e)$ is uniquely determined and we say that $\left(\Sigma_{b}, C\right)$ has the degree $(\sigma, e)$. Moreover, let $m_{1}$ denote the highest multiplicity of singular points of $C$. The pair $\left(\Sigma_{b}, C\right)$ is said to be \#-minimal if $\sigma \geq 2 m_{1}$ and $e-\sigma \geq b m_{1}$ (cf. [I1]). The last condition is always satisfied whenever $b \geq 2$.

Let $D$ be a non-singular curve on $S$. Then the pair $(S, D)$ is said to be relatively minimal, whenever the intersection number $D \cdot E \geq 2$ for any exceptional curve $E$ of the first kind on $S$ such that $E \neq D$ (cf. [I1], Theorem 1, [S]). Suppose that $(S, D)$ is a relatively minimal pair such that $S \neq \mathbf{P}^{2}$ and $\kappa[D] \geq 0$ where $\kappa[D]$ denotes $\kappa(K+D, S)$. Then there exists a \#-minimal pair $\left(\Sigma_{b}, C\right)$ such that $(S, D)$ is derived from $\left(\Sigma_{b}, C\right)$ by resolving singularities on $C$ in a shortest way (cf. [I1]). In this case, we say that $\left(\Sigma_{b}, C\right)$ is a \#-minimal model of $(S, D)$. The structure of $(S, D)$ with $\kappa[D] \leq 1$ has been precisely determined by litaka in [I1] and [I2]. If $\kappa[D]=2$, then relatively minimal pairs are always minimal (see [I1]), and hence, $D^{2}$ is invariant for birational transformation of pairs. Note that if $\kappa[D] \geq 0$, then $\sigma$

Received November 5, 1999

Revised March 15, 2001

The result of the paper is part of the doctoral thesis submitted to Gakushuin University, on December 24, 1998. 
is also invariant for birational transformations of pairs (see [I2]). Define an invariant $n$ to be $4 g-D^{2}$ where $g$ is the genus of $D$. If $\kappa[D]=2$ then $n \geq-4$. Moreover, if $\kappa[D]=2$ and $n \leq 4$ then $\sigma \leq 4$ ([I2, Proposition 7]).

The main result is stated as follows:

THEOREM 1. Let $(S, D)$ be a pair of a rational surface $S$ and a non-singular curve $D$ of genus $g$ on $S$. Suppose that $(S, D)$ is a relatively minimal pair and $S \neq \mathbf{P}^{2}, \kappa[D]=2$. By $(\sigma, e)$, we denote the degree of a \#-minimal model $\left(\Sigma_{b}, C\right)$ of $(S, D)$. Then $\sigma \leq(n-1)(n-2)$ for $n \geq 5$.

Furthermore, all possible types of relatively minimal pairs for $5 \leq n \leq 6$ will be enumerated in Theorem 2.

The author expresses gratitude to Professors Shigeru Iitaka and Kazuo Akao for their valuable advices during the preparation of this paper. Moreover, the author would like to thank the referee for his very valuable suggestions which are essential to complete the final draft.

\section{Proof of Theorem 1.}

Let $(S, D)$ be a relatively minimal pair such that $S \neq \mathbf{P}^{2}$ and $\kappa[D]=2$. Then there exists a \#-minimal model of $(S, D)$, which is denoted by $\left(\Sigma_{b}, C\right)$. After a finite succession of blowing ups at singular points of $C$, we have a birational morphism $\mu: S \rightarrow \Sigma_{b}$. Let $P_{1}, P_{2}, \ldots, P_{r}$ indicate all the singular points (including infinitely near singular points) of $C$ whose multiplicities are denoted by $m_{1}, m_{2}, \ldots, m_{r}$, respectively. Here it is supposed that $m_{1} \geq \cdots \geq m_{r}$. Then we have the following relations:

$$
\begin{aligned}
& K \sim \mu^{*}\left(K_{0}\right)+\sum_{j=1}^{r} E_{j}, \\
& D \sim \mu^{*}(C)-\sum_{j=1}^{r} m_{j} E_{j} .
\end{aligned}
$$

Here, the $E_{j}$ are exceptional curves of the first kind derived from blowing up singular points $P_{j}$ and for simplicity, the total inverse images of $E_{j}$ are denoted by the same symbols $E_{j} . K_{0}$ and $K$ mean canonical divisors on $\Sigma_{b}$ and $S$, respectively. Furthermore, letting $(\sigma, e)$ be the degree of $\left(\Sigma_{b}, C\right)$, we obtain

$$
\begin{aligned}
C^{2} & =2 e \sigma-\sigma^{2} b, \\
\pi & =(e-1)(\sigma-1)-\frac{b \sigma(\sigma-1)}{2}
\end{aligned}
$$

where $\pi$ denotes the virtual genus of $C$. From these, we have the following equations:

$$
D^{2}=C^{2}-\sum_{j=1}^{r} m_{j}^{2}=2 e \sigma-\sigma^{2} b-\sum_{j=1}^{r} m_{j}^{2},
$$




$$
g=\pi-\sum_{j=1}^{r} \frac{m_{j}\left(m_{j}-1\right)}{2}=(e-1)(\sigma-1)-\frac{b \sigma(\sigma-1)}{2}-\sum_{j=1}^{r} \frac{m_{j}\left(m_{j}-1\right)}{2} .
$$

(I) Case $r=0$ : Then $C$ is non-singular; hence $C=D$. We have $n=4 g-D^{2}=$ $4 \pi-C^{2}$. From the Equations (3) and (4), we obtain

$$
(\sigma-2)(2 e-\sigma b-4)=n+4 .
$$

Hence, it follows that $\sigma-2 \leq n+4$. Thus, $\sigma \leq n+6$, which implies $\sigma<(n-1)(n-2)$ since $n \geq 5$.

(II) Case $r \geq 1$ and $b \geq 3$ : Define $H$ to be $2(\pi-h) \sigma-(\sigma-2) C^{2}$ where $h=[(n+1) / 2]$ (cf. [H2, p. 117]). Since $\sigma \geq 2 m_{1}$,

$$
2(g-h) \sigma-(\sigma-2) D^{2}=H+\sum_{j=1}^{r} m_{j}\left(\sigma-2 m_{j}\right) \geq H .
$$

Suppose that $H \geq 0$. Then

$$
-n \leq g-n=D^{2}-3 g \leq D^{2}-\frac{2 \sigma}{\sigma-2} g \leq-\frac{2 h \sigma}{\sigma-2}<-2 h .
$$

Hence, $h<n / 2$, which contradicts the inequality $h=[(n+1) / 2] \geq n / 2$. Hence, $H<0$; thus,

$$
2\{e-(b \sigma+h-1)\}+\sigma(b-2)=H / \sigma<0 .
$$

From this, it follows that

$$
\sigma(b-2)<-2\{e-(b \sigma+h-1)\} \leq 2(h-1) .
$$

Since $b \geq 3$, we have $\sigma<2(h-1) \leq(n-1)$. Hence, $\sigma<(n-1)(n-2)$.

(III) Case $r \geq 1$ and $b \leq 2$ : By (5) and (6),

$$
\begin{aligned}
& \sum_{j=1}^{r} m_{j}=\sigma(2 e-\sigma b)-(\sigma-1)(2 e-\sigma b-2)+n-2 g, \\
& \sum_{j=1}^{r} m_{j}^{2}=\sigma(2 e-\sigma b)+n-4 g .
\end{aligned}
$$

If $b=0$, then put $\sigma=2 m_{1}+v$ and $e=\sigma+u$. If $b=1$, then put $\sigma=2 m_{1}+v$ and $e=\sigma+m_{1}+u$. If $b=2$, then put $\sigma=2 m_{1}+v$ and $e=2 \sigma+u$. Note that in these cases $u \geq 0$ and $v \geq 0$. The Equations (10) and (11) turn out to be

$$
\begin{aligned}
& \sum_{j=1}^{r} m_{j}=8 m_{1}+2 u+w v+n-2 g-2 \\
& \sum_{j=1}^{r} m_{j}^{2}=8 m_{1}^{2}+2(2 u+w v) m_{1}+(w-2) v^{2}+2 u v+n-4 g .
\end{aligned}
$$


Here, we put $w=4-\delta_{1, b}$ where $\delta_{i, j}$ denotes the Kronecker delta. By (12), (13) and the following inequality

$$
m_{1} \sum_{j=1}^{r} m_{j}-\sum_{j=1}^{r} m_{j}^{2} \geq 0
$$

we have

$$
(n-2 g-2-2 u-w v) m_{1} \geq(w-2) v^{2}+2 u v+n-4 g .
$$

Note that if $g=0$, then $n-2 g-2-2 u-w v>0$ by (14).

(III-1) Suppose that $n-2 g-2-2 u-w v<0$. Then by (14) and $g \geq 1$,

$$
m_{1} \leq \frac{4 g-n-(w-2) v^{2}-2 u v}{2 g+2-n+2 u+w v} \leq \frac{4 g-n}{2 g+2-n} \leq 2+\frac{n-4}{2 g+2-n} \leq 1 .
$$

This contradicts the hypothesis $m_{1} \geq 2$.

(III-2) Suppose that $n-2 g-2-2 u-w v \geq 0$. Then we obtain

$$
n-2 \geq 2 u+w v+2 g \text {. }
$$

LEMMA 1. Given non-negative integers $x_{1}, \ldots, x_{l}, m, \alpha$ and $\beta$ such that $\sum_{j=1}^{l} x_{j}=$ $\alpha m+\beta$ and $x_{j} \leq m$ for $1 \leq j \leq l$, it follows that $\sum_{j=1}^{l} x_{j}^{2} \leq \alpha m^{2}+\beta^{2}$.

PROOF. First we consider the case where $\alpha$ is the quotient $q$ of $\sum_{j=1}^{r} x_{j}$ by $m$ and $\beta$ is the remainder $\gamma$. We prove this by induction on $l$. When $l=1$, the result follows immediately. In the case where $l \geq 2$, if there exist $i \neq j$ such that $x_{i}+x_{j}<m$, then by induction hypothesis,

$$
\sum_{j=1}^{l} x_{j}^{2} \leq\left(x_{i}+x_{j}\right)^{2}+\sum_{k \neq i, j} x_{k}^{2} \leq q m^{2}+\gamma^{2} .
$$

Suppose that there exist $i \neq j$ such that $x_{i}+x_{j} \geq m$. Since $x_{i} \leq m$ and $x_{j} \leq m$, it follows that $x_{i}+x_{j}-m \leq m$ and $m^{2}+\left(x_{i}+x_{j}-m\right)^{2}=x_{i}^{2}+x_{j}^{2}+2\left(m-x_{i}\right)\left(m-x_{j}\right) \geq x_{i}^{2}+z_{j}^{2}$. Thus,

$$
\sum_{j=1}^{l} x_{j}^{2}=x_{i}^{2}+x_{j}^{2}+\sum_{k \neq i, j} x_{k}^{2} \leq m^{2}+\left(x_{i}+x_{j}-m\right)^{2}+\sum_{k \neq i, j} x_{k}^{2} .
$$

By induction hypothesis,

$$
\left(x_{i}+x_{j}-m\right)^{2}+\sum_{k \neq i, j} x_{k}^{2} \leq q^{\prime} m^{2}+\gamma^{2}
$$

where $q^{\prime}$ is the quotient of $\left(x_{i}+x_{j}-m\right)+\sum_{k \neq i, j} x_{k}$ by $m$ and $\gamma$ is the remainder. Putting $q=q^{\prime}+1$, we have the result.

Next consider the general case. Letting $q$ denote the quotient of $\alpha m+\beta$ by $m$ and $\gamma$ the remainder, we have $\alpha m+\beta=q m+\gamma$ and $q=\alpha+\alpha^{\prime}$ for some $\alpha^{\prime} \geq 0$. Hence, $\beta=\gamma+\alpha^{\prime} m$. 
By the former part of the proof, we obtain

$$
\sum_{j=1}^{l} \leq q m^{2}+\gamma^{2}
$$

From

$$
q m^{2}+\gamma^{2}=\left(\alpha+\alpha^{\prime}\right) m^{2}+\gamma^{2} \leq \alpha m^{2}+\left(\alpha^{\prime} m+\gamma\right)^{2}=\alpha m^{2}+\beta^{2},
$$

the result follows immediately.

Note: The author thanks Dr. H. Tanaka for his advice in the proof.

Applying Lemma 1 to (12) and (13) where $\alpha=8, m=m_{1}$ and $\beta=2 u+w v+n-2 g-2$, we have

$$
8 m_{1}^{2}+2(2 u+w v) m_{1}+(w-2) v^{2}+2 u v+n-4 g=\sum_{j=1}^{r} m_{j}^{2} \leq 8 m_{1}^{2}+(2 u+w v+n-2 g-2)^{2} .
$$

From this, it follows that

$$
2(2 u+w v) m_{1}+(w-2) v^{2}+2 u v+n-4 g \leq(2 u+w v+n-2 g-2)^{2} .
$$

Recalling that $\sigma=2 m_{1}+v$, we obtain

$$
(2 u+w v)(\sigma-v)+(w-2) v^{2}+2 u v+n-4 g \leq(2 u+w v+n-2 g-2)^{2},
$$

and then

$$
(2 u+w v) \sigma \leq(2 u+w v+n-2)^{2}-n+2 v^{2}-4 g(2 u+w v+n-g-3) .
$$

By (15), we have $2 g \leq n-2$ and so $-4 g(2 u+w v+n-g-3) \leq 0$. Thus, we obtain

$$
(2 u+w v) \sigma \leq(2 u+w v+n-2)^{2}-n+2 v^{2} .
$$

Case $2 u+w v>0$ : By (15), we have $2 \leq 2 u+w v \leq 2 u+w v+2 g \leq n-2$ and so $v \leq(n-2) / w \leq(n-2) / 3$. Hence,

$$
\begin{aligned}
\sigma & \leq \frac{(2 u+w v+n-2)^{2}-n+2 v^{2}}{2 u+w v} \\
& =2 u+w v+2(n-2)+\frac{(n-2)^{2}-n+2 v^{2}}{2 u+w v} \\
& \leq 3(n-2)+\frac{1}{2}\left\{(n-2)^{2}-n+2 v^{2}\right\} \\
& \leq \frac{1}{2}\left\{(n-1)(n-2)+4 n-10+2(n-2)^{2} / 9\right\} \\
& =(n-1)(n-2)+\frac{1}{2}\{(n-2)(-7 n+41) / 9-2\} .
\end{aligned}
$$

Here, $(-7 n+41)(n-2) / 9-2 \leq 0$ for $n \geq 5$. Here, $\sigma \leq(n-1)(n-2)$. 
Case $2 u+w v=0$ : Then $u=v=0$. Hence, $\sigma=2 m_{1}$. Define $t$ to be $\max \left\{j \mid m_{1}=\right.$ $\left.m_{j}\right\}$. Then (12) and (13) turn out to be

$$
\begin{aligned}
& t m_{1}+\sum_{j=t+1}^{r} m_{j}=8 m_{1}+n-2 g-2, \\
& t m_{1}^{2}+\sum_{j=t+1}^{r} m_{j}^{2}=8 m_{1}^{2}+n-4 g .
\end{aligned}
$$

Here, $m_{j} \leq m_{1}-1$ for $j \geq t+1$.

Suppose that $t \geq 8$. Eliminating $n$ from (16) and (17), we have

$$
0 \leq(t-8)\left(m_{1}^{2}-m_{1}\right)+\sum_{j=9}^{r}\left(m_{j}^{2}-m_{j}\right)=2-2 g \leq 2 .
$$

Hence, $g=0$ or $g=1$. If $g=0$, then $t=8, r=9$ and $m_{9}=2$. If $g=1$, then $t=r=8$. In both cases, making use of (16) again, we can verify $n=4$, which contradict the hypothesis $n \geq 5$.

Suppose that $1 \leq t \leq 7$. Then the Equations (16) and (17) imply that

$$
\begin{aligned}
& \sum_{j=t+1}^{r} m_{j}+2 g=(8-t) m_{1}+n-2=(8-t)\left(m_{1}-1\right)+n+6-t, \\
& \sum_{j=t+1}^{r} m_{j}^{2}+4 g^{2}=(8-t) m_{1}^{2}+n-4 g+4 g^{2} .
\end{aligned}
$$

Here, $n+6-t>0$ by $1 \leq t \leq 7$. By applying Lemma 1 where $m=m_{1}-1, \alpha=8-t$ and $\beta=n+6-t$, we obtain

$$
(8-t) m_{1}^{2}+n-4 g+4 g^{2} \leq(8-t)\left(m_{1}-1\right)^{2}+(n+6-t)^{2} .
$$

From this, it follows that

$$
2(8-t) m_{1} \leq(8-t)+(n+6-t)^{2}-n-4 g(g-1) .
$$

Since $4 g(g-1) \geq 0$, we have

$$
\begin{aligned}
2(8-t) m_{1} & \leq(8-t)+(n-2+8-t)^{2}-n \\
& =(8-t)+(n-2+8-t)^{2}-n \\
& =(8-t)(2 n+5-t)+n^{2}-5 n+4 \\
& =(8-t)(n-1)(n-2)+(8-t)\left(-n^{2}+5 n+3-t\right)+n^{2}-5 n+4 \\
& =(8-t)(n-1)(n-2)-(7-t)\left(n^{2}-5 n+t-4\right) .
\end{aligned}
$$

Thus,

$$
m_{1} \leq \frac{1}{2}\left\{(n-1)(n-2)-\left(n^{2}-5 n+t-4\right)(7-t) /(8-t)\right\}
$$


However, since $t \leq 7$, we have $-\left(n^{2}-5 n+t-4\right)(7-t) /(8-t)<2$ unless $t=1$ and $n=5$. Hence, we have $m_{1} \leq(n-1)(n-2) / 2$, and so $\sigma=2 m_{1} \leq(n-1)(n-2)$. Finally, we consider the case where $n=5$ and $t=1$. Then $g \leq n-4=1$ by Proposition 2. By (18), we have $m_{1} \leq 7$. If $m_{1} \leq 6$ then $\sigma=2 m_{1} \leq 12$ and $\sigma \leq(n-1)(n-2)=12$. Therefore, in order to complete the proof, it suffices to derive a contradiction under the hypothesis that $t=1$ and $m_{1}=7$. Then in these case, $m_{2} \leq 6$. (16) and (17) turn out to be

$$
\sum_{j=2}^{r} m_{j}=52-2 g, \quad \sum_{j=2}^{r} m_{j}^{2}=348-4 g .
$$

Then

$$
(52-2 g) m_{2}-348+4 g=m_{2} \sum_{j=2}^{r} m_{j}-\sum_{j=2}^{r} m_{j}^{2} \geq 0 .
$$

Since $g=0$ or $g=1$, it follows that $m_{2} \geq(348-4 g) /(52-2 g)>6$, which contradicts the inequality $m_{2} \leq 6$. This completes the proof of Theorem 1 .

\section{Types of \#-minimal models of $(S, D)$.}

All possible types of \#-minimal models of relatively minimal pairs $(S, D)$ with $\kappa[D]=2$ and $n=4 g-D^{2} \geq 5$ can be enumerated in the following way.

Let $\left(\Sigma_{b}, C\right)$ be a \#-minimal pair with degree $(\sigma, e)$. Furthermore, let $m_{1}, m_{2}, \ldots, m_{r}$ be the multiplicities of all singular points $P_{1}, P_{2}, \ldots, P_{r}$ of $C$ where $m_{1} \geq m_{2} \geq \cdots \geq m_{r}$.

Case (A). Case $C=D$ : By the Equation (7), it is easy to see that $\sigma \leq n+4, b \leq$ $(n+8) / 2$ and $2 e \leq n+8+\sigma b$. The set of solutions $(\sigma, e, b)$ satisfying the above equality and inequalities is finite. So, we are done.

Case (B). Case in which $C$ is singular:

Proposition 2. Suppose that $g \geq 1$ and $C$ is singular. Then $g \leq n+5-r$.

Proof. Since $D+K$ is nef and $\left|D+m_{1} K\right|$ is not empty ([12, Proposition 3]), $(D+$ $K)\left(D+m_{1} K\right) \geq 0$. From the adjunction formula,

$$
K^{2} \geq-\frac{2 g-2}{m_{1}}-2 g+2+D^{2} \geq-3 g+3+D^{2} \geq g+3-n .
$$

Since $K^{2}=8-r$, we obtain $g \leq n+5-r$.

Proposition 3. Suppose that $g=0$ and $n=-D^{2} \geq 5$. Then $-(n-2)^{2} / n \leq K^{2} \leq$ -1 .

Proof. We have $(D+n /(n-2) K) \cdot\left(D+m_{1} K\right) \geq 0$, since $D+n /(n-2) K$ is nef and $\left|D+m_{1} K\right|$ is not empty ([I2, Proposition 3]). Hence, $(D+n /(n-2) K) \cdot K \geq 0$, which induces $K^{2} \geq-(n-2)^{2} / n$. Supposing that $K^{2} \geq 0$, we shall derive a contradiction. Then we have $|-K| \neq \phi$ by Riemann-Roch theorem. Thus, $(D+n /(n-2) K) \cdot(-K) \geq 0$; hence, $(D+n /(n-2) K) \cdot K=0$. Therefore, $K^{2}=-(n-2)^{2} n=-n+4-4 / n$, which is not an integer, since $n \geq 5$. Thus $K^{2} \leq-1$ is established. 
In the case $g=0$, Proposition 3 and $K^{2}=8-r$ imply $9 \leq r \leq n+4$. If $g \geq 1$, then by Proposition 2 and $r \geq 1$, we have $g \leq n+4$ and $r \leq n+4$.

(B-1) Case $b \geq 3$ : Then by (9), $\sigma<2(h-1)$ and $b<2(h-1) / \sigma+2$ where $h=[(n+1) / 2]$. Moreover, by the Equation (8), we have $e<(b \sigma+h-1)-\sigma(b-2) / 2$.

(B-2) Case $b \leq 2$ : Then $e=2 \sigma+u$ where $u<(n-2) / 2-2 g$ by (15). Hence, $e<2 \sigma+(n-2) / 2-2 g$.

Theorem 1 gives an upper bound for $\sigma$. Accordingly, since $\sigma \geq 2 m_{1}$ and $m_{1} \geq m_{2} \geq$ $\cdots \geq m_{r}$, all possible types of \#-minimal models $\left(\Sigma_{b}, C\right)$ could be computed.

We say that the symbol $\left[\sigma * e, b ; m_{1}, \ldots, m_{r}\right]$ is the type of $\left(\Sigma_{b}, C\right)$. For simplicity, $\left[\sigma * e, 0 ; m_{1}, \ldots, m_{r}\right]$ is rewritten as $\left[\sigma * e ; m_{1}, \ldots, m_{r}\right]$. Furthermore, when $\sigma=2 m_{1}$, we say that both $\left[\sigma *\left(e+m_{1}\right), 1 ; m_{1}, \ldots, m_{r}\right]$ and $\left[\sigma *\left(e+2 m_{1}\right), 2 ; m_{1}, \ldots, m_{r}\right]$ are types associated to the type $\left[\sigma * e ; m_{1}, \ldots, m_{r}\right]$.

THEOREM 2. Let $(S, D)$ be a relatively minimal pair. Suppose that $5 \leq n \leq 6$ where $n=4 g-D^{2}$. Then all possible types of \#-minimal models of $(S, D)$ are listed in the following table.

\begin{tabular}{r|r|r|l}
\hline \multicolumn{1}{r|}{$n$} & \multicolumn{1}{c|}{$g$} & \multicolumn{1}{c|}{$r$} & types of \#-minimal pairs of $(S, D)$ \\
\hline \multirow{2}{*}{5} & 0 & 9 & {$\left[10 * 11 ; 5^{9}\right],\left[12 * 12 ; 6^{7}, 5,4\right]$ and types associated to these. } \\
\cline { 2 - 4 } & 10 & 0 & {$[5 * 6,1 ; 1]$} \\
\cline { 2 - 4 } & 11 & 0 & {$[3 * 8,1 ; 1],[3 * 11,3 ; 1]$} \\
\hline \multirow{2}{*}{0} & 0 & 9 & $\begin{array}{l}{\left[16 * 16 ; 8^{6}, 7^{2}, 6\right],\left[20 * 20 ; 10^{7}, 9,5\right] \text { and types associated to these, }} \\
\text { and }\left[15 * 22,1 ; 7^{9}\right]\end{array}$ \\
\cline { 2 - 4 } & & 10 & {$\left[6 * 7 ; 3^{10}\right],\left[8 * 8 ; 4^{7}, 3^{2}, 2\right]$ and types associated to these. } \\
\cline { 2 - 4 } & 1 & 9 & {$\left[8 * 8 ; 4^{7}, 3^{2}\right]$ and types associated to these. } \\
\cline { 2 - 4 } & 12 & 0 & {$[3 * 7 ; 1],[3 * 10,2 ; 1],[3 * 13,4 ; 1]$} \\
\hline
\end{tabular}

The author does not know concrete curves which have the above types. However, he has obtained the table of similar results even in the case where $n=7,8,9,10$ with help of computer (cf. [M]).

\section{References}

[H1] R. HARTSHORNE, Algebraic Geometry, Springer (1977).

[H2] R. HARTSHORNE, Curves with high self-intersection on algebraic surfaces, Publ. I.H.E.S. 36 (1970), 111126.

[I1] S. IITAKA, On irreducible plane curves, Saitama Math. J. 1 (1983), 47-63.

[I2] S. IITAKA, Birational geometry of plane curves, Tokyo J. Math. 22 (1999), 289-321.

[S] F. SAKAI, Semi-stable curve of algebraic surfaces and logarithmic pluricanonical maps, Math. Ann. 254 (1980), 89-120. 
[M] O. MATSUDA, Birational geometry of curves on rational and ruled surfaces, Doctoral Thesis, Gakushuin Univ. (1998).

Present Address:

TSUyama National College of Technology, NUMA, TSUYAMA, OKAYAMA, 708-8509 JAPAN.

e-mail: matsuda@tsuyama-ct.ac.jp 